\title{
Variáveis de copa na determinação da densidade máxima de florestas de Araucaria angustifolia (Bertol) Kuntze
}

\author{
Crown variables in the determination of the maximum density of Araucaria \\ angustifolia (Bertol) Kuntze forests
}

\author{
Ana Claudia da Silveira ${ }^{1}$ (D), Andre Felipe Hess ${ }^{1}$ (D), Luis Paulo Baldissera Schorr ${ }^{2}$ (D), \\ Thiago Floriani Stepka ${ }^{1}$ (D), Sandra Mara Krefta ${ }^{1}$ (D), Kemely Alves Atanazio ${ }^{3}$ (D) \\ ${ }^{1}$ Universidade do Estado de Santa Catarina - UDESC, Lages, SC, Brasil \\ ${ }^{2}$ Universidade Federal de Lavras - UFLA, Lavras, MG, Brasil \\ ${ }^{3}$ Universidade Federal de Viçosa - UFV, Viçosa, MG, Brasil
}

\begin{abstract}
Como citar: Silveira, A. C., Hess, A. F., Schorr, L. P. B., Stepka, T. F., Krefta, S. M., \& Atanazio, K. A. (2021). Variáveis de copa na determinação da densidade máxima de florestas de Araucaria angustifolia (Bertol) Kuntze. Scientia Forestalis, 49(130), e3545. https://doi.org/10.18671/scifor.v49n130.16
\end{abstract}

\begin{abstract}
Resumo
A ocupação do espaço individual máximo com base no tamanho da copa é um critério coerente para a definição do momento ideal para realizar intervenções na floresta. O objetivo do trabalho foi gerar equações entre a densidade máxima de árvores por hectare e as variáveis de copa, visando propor intervenções silviculturais. $O$ estudo foi realizado em uma floresta nativa com 84 hectares localizada no município de Lages, SC. Os dados foram medidos de 25 parcelas permanentes de $400 \mathrm{~m}^{2}$, onde todas as árvores com diâmetro à altura do peito (dap) $\geq 10 \mathrm{~cm}$ foram mensuradas quanto ao dap, a altura total, a altura de inserção de copa e quatro raios de copa. Com o conjunto de dados foi calculado o diâmetro de copa, comprimento de copa, o fator de competição de copa, a área de superfície de copa, o volume de copa e número de árvores por hectare. A média de Fator de Competição de Copa foi superior a 95\% evidenciando um alto grau de cobertura do solo pelas copas e fechamento do dossel. Esse resultado dificulta a regeneração da espécie e crescimento de árvores individuais, caracterizando a necessidade de intervenção silvicultural. A densidade máxima por hectare considerando a área de superfície de copa e o volume de copa foi de 171 árvores para um diâmetro médio de $32 \mathrm{~cm}$.
\end{abstract}

Palavras-chave: Manejo floresta; Morfometria; Mensuração florestal.

\begin{abstract}
Occupancy of the maximum individual space based on crown size is a coherent criterion for defining the optimal time to perform the forest intervention. This work aimed to generate equations between maximum tree density per hectare and the crown variables, allowing to suggest a silvicultural intervention and forest management. The study was carried out in a native forest of 84 hectares and it is located in the municipality of Lages, SC. Data were measured in 25 permanent plots of $400 \mathrm{~m}^{2}$, where all trees with diameter at breast height (dap) greater than $10 \mathrm{~cm}$ were measured regarding the dap, total height, height of crown insertion and four crown radii. With the data set we calculated the crown diameter, the crown length, the crown competition factor, the crown surface area, the crown volume and the number of trees per hectare. The mean of the Crown Competition Factor was greater than 95\%, and it evidences the high degree of crown cover and crown closure. Such a percentage value hinders the species regeneration and the individual tree growth, characterizing the need for silvicultural intervention. The maximum density per hectare, considering a crown surface area and crown volume, was 171 trees for a mean diameter of $32 \mathrm{~cm}$.
\end{abstract}

Keywords: Forest management; Morphometry; Forest measurement.

Fonte de financiamento: Nenhuma.

Conflito de interesse: Nada a declarar.

Autor correspondente: Ipbs93@gmail.com

Recebido: 20 maio 2020.

Aceito: 21 agosto 2020.

Editor: Paulo Henrique Müller Silva.

(c) (i) Este é um artigo publicado em acesso aberto (Open Access) sob a licença Creative Commons Attribution, que permite uso, distribuição e

c) reprodução em qualquer meio, sem restrições desde que o trabalho original seja corretamente citado. 


\section{INTRODUÇÃO}

Atualmente as florestas de Araucária do sul do Brasil apresentam-se superestocadas (Hess et al., 2018a), contendo um baixo número de indivíduos de Araucaria angustifolia (Bertol) Kuntze nos estágios iniciais de desenvolvimento (Narvaes et al., 2005; Kanieski et al., 2012), bem como, um baixo ingresso de indivíduos para classes de porte adulto (Silveira et al., 2018).

Diante do panorama atual das florestas de Araucária, cresce a preocupação sobre o futuro desses remanescentes florestais, uma vez que a elevada competição existente entre as árvores tem efeitos negativo sobre a dinâmica estrutural e diversidade de espécies (Schorr, 2019). Portanto, efetiva-se a importância da determinação das dimensões das árvores para fins de manejo florestal e regulação da densidade ideal dessas florestas (Hess et al., 2018c).

Sabe-se que determinar o espaço horizontal nas florestas e também a sua relação com as dimensões das árvores é objeto de estudo de vários pesquisadores (Pretzsch, 2014, Klein et al., 2017; MacFarlane \& Kane, 2017; Hess et al., 2018a), considerando principalmente as relações estabelecidas entre as variáveis: diâmetro de copa, altura e diâmetro da árvore e diâmetro e comprimento de copa, as quais são de grande relevância nos estudos de competição em povoamentos florestais.

O conhecimento dos efeitos da competição aliado ao da densidade máxima que o sítio florestal pode suportar são imprescindíveis para o bom gerenciamento florestal (Meyer et al., 2013), uma vez que essas informações auxiliam no planejamento de intervenções silviculturais que buscam favorecer o desenvolvimento de toda floresta, controlando a mortalidade.

A partir dessas afirmações, esse trabalho considera a hipótese de que a ocupação do espaço individual máximo com base no tamanho da copa é um critério coerente para a definição do momento ideal de realizar intervenções na floresta. Sendo assim, o objetivo do trabalho consiste em gerar equações que descrevam a relação da densidade máxima de árvores por hectare e as variáveis de copa para serem utilizadas como subsídios de manejo florestal sustentável para a realização de intervenções silviculturais em uma floresta de Araucária localizada em Lages, SC.

\section{MATERIAL E MÉTODOS}

\section{Caracterização da área de estudo}

O estudo foi realizado em um remanescente de Araucaria angustifolia, com área total de 84 ha, situado nas coordenadas de $27^{\circ} 49^{\prime} \mathrm{S}$ e $50^{\circ} 06^{\prime} \mathrm{O}$, no município de Lages - SC. Segundo a classificação de Köppen o clima é Cfb, ou seja, subtropical úmido, sem estação seca definida. A temperatura média anual é de $15,2^{\circ} \mathrm{C}$, a precipitação média anual é de $1684,7 \mathrm{~mm}$ e a altitude média é de $987,0 \mathrm{~m}$ (Alvares et al., 2013).

Este remanescente apresenta 330 árvores de araucária por hectare, sendo que as mesmas estão distribuídas em classes de diâmetro que variam de 10 a $70 \mathrm{~cm}$. 0 diâmetro médio das árvores é de $29 \mathrm{~cm}$, com altura média de $16,2 \mathrm{~m}$ e média de $5,5 \mathrm{~m}$ para o diâmetro de copa (Hess et al., 2018c).

\section{Coleta dos dados}

Os dados são provenientes de vinte e cinco (25) parcelas com dimensão de $20 \times 20 \mathrm{~m}$ $\left(400 \mathrm{~m}^{2}\right)$. Para alocação das parcelas foi utilizado o método de amostragem sistemático, considerando uma distância entre parcelas de 50 m e entre linhas de 100 m (Figura 1). 


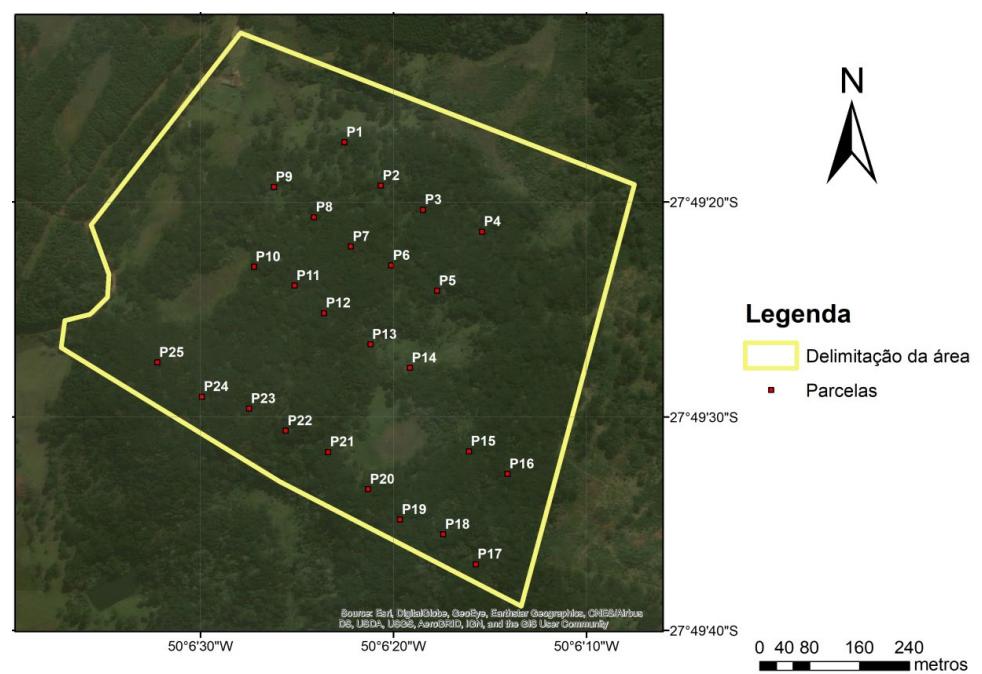

Figura 1. Imagem aérea da área de estudo e distribuição das parcelas avaliadas.

Em cada uma das parcelas, todas as árvores de araucária que apresentaram diâmetro à altura do peito (DAP) $\geq 10 \mathrm{~cm}$ foram mensuradas quanto ao DAP, altura total $(\mathrm{H})$, altura de início de copa (HIC) e 4 raios de copa (RC) considerando o sentido de cada ponto cardeal: Norte, Leste, Sul e Oeste. Para a mensuração do DAP foi utilizada suta dendrométrica, enquanto para H, HIC e RC utilizou-se hipsômetro laser TruPulse.

Posteriormente, para obtenção do raio médio de copa (calculou-se a média entre os 4 raios de copa. A partir disso, calcularam-se as variáveis de copa, duplicando o valor do raio médio da copa:

$D C=\overline{r c m} * 2$

Em que: DC o diâmetro de copa $(\mathrm{m})$ e $\overline{r c m}$ o raio médio de copa (m).

Já com os valores de altura total e altura de início de copa, calculou-se o comprimento de copa (CC):

$C C=H-H I C$

Em que: CC o comprimento de copa (m), H a altura total (m) e HIC a altura de início de copa (m).

\section{Correlação das variáveis}

Complementarmente, as variáveis dendro-morfométricas (DAP, H, HIC, DC, CC) foram submetidas a análise de correlação de Pearson a um nível de significância de 0,05. A análise dos dados foi realizada com auxílio do software Statistical Analysis System - SAS 9.3.

\section{Competição das copas}

O Fator de Competição da Copa (FCC) foi calculado considerando as estimativas das áreas de copa das árvores que compõe o povoamento, se essas tivessem o mesmo diâmetro de copa das árvores crescendo livres de competição (Thomas, 2009).

Para a obtenção do diâmetro de copa livre de competição (DCL), utilizou-se um modelo linear, onde o DAP foi considerando como variável independente. Essa modelagem considerou apenas os valores de árvores que crescem isoladas e que não estão sobre competição. O ajuste foi realizado no software Statistical Analysis System - SAS 9.3, sendo analisados os critérios estatísticos do coeficiente de determinação $\left(R^{2}\right)$ e o erro padrão de estimativa (Syx\%). 
em que: DCL o diâmetro de copa Livre $(\mathrm{m})$, os parâmetros do modelo e DAP o diâmetro à altura do peito $(\mathrm{cm})$.

Através do diâmetro de copa livre estimado, calculou-se a Área de copa livre (ACL) utilizando a equação

$A C L=\frac{\pi^{*} D C L^{2}}{4}$

em que: $A C L$ a área de copa livre $\left(m^{2}\right)$ e $D C L$ o Diâmetro de copa livre $(m)$.

Após obter a área de copa livre foi possível calcular o FCC através da equação:

$F C C=\frac{\sum A C L}{S} * 100$

em que: FCC o fator de competição de copa, $\mathrm{ACL}$ a área de copa livre na unidade amostral $\left(\mathrm{m}^{2}\right)$ e $\mathrm{S}$ a área da unidade amostral $\left(\mathrm{m}^{2}\right)$.

\section{Determinação da densidade máxima por hectare}

Considerando o diâmetro de copa e comprimento da copa, pode-se calcular a Área de Superfície de Copa (ASC) e o Volume de Copa, assumindo que a copa é um sólido geométrico regular na forma de conóide. Para o cálculo da ASC foi utilizada a equação abaixo, citada por Burkhart \& Tomé (2012).

$$
A S C=\frac{\pi D C}{2}{\sqrt{C C^{2}+\left(\frac{D C}{2}\right)^{2}}}^{2}
$$

em que: ASC a área de superfície de copa $\left(\mathrm{m}^{2}\right)$, DC o diâmetro de copa $\left(\mathrm{m}^{2}\right)$ e CC o comprimento de copa (m).

E para o volume de copa utilizou-se a Equação descrita por Burkhart \& Tomé (2012).

$V C=\frac{\pi^{*} D C^{*} C C}{12}$

em que: VC o volume de copa $\left(\mathrm{m}^{3}\right)$, DC o diâmetro de copa (m) e CC o comprimento de copa (m).

Assim o número de árvores por hectare, através da ocupação das copas foi estabelecido em função da equação:

$N / h a=\frac{10000}{A S C}$

em que: N/ha o número de árvores por hectare (arv/ha) e ASC a área de superfície de copa $\left(\mathrm{m}^{2}\right)$. 


\section{RESULTADOS E DISCUSSÃO}

\section{Correlação das variáveis}

As variáveis mensuradas a campo (DAP, H, HIC), juntamente com o diâmetro de copa (DC) e o comprimento de copa (CC) foram correlacionadas e o resultado pode-se observar na Tabela 1.

Tabela 1. Valores da Correlação de Pearson estabelecida entre as variáveis dendrométricas e morfométricas para Araucaria angustifolia em Lages, SC.

\begin{tabular}{cccccc}
\hline & DAP & H & HIC & DC & CC \\
\hline DAP & 1 & - & - & - & - \\
H & 0,73787 & 1 & - & - & - \\
HIC & 0,49272 & 0,80787 & 1 & - & - \\
DC & 0,80239 & 0,54889 & 0,33099 & 1 & - \\
CC & 0,51941 & 0,49776 & $-0,10904$ & 0,46868 & 1 \\
\hline
\end{tabular}

em que: DAP o diâmetro à altura do peito $(\mathrm{cm}), \mathrm{H}$ a altura total $(\mathrm{m}), \mathrm{HIC}$ a altura de início de copa $(\mathrm{m}), \mathrm{DC}$ o diâmetro de copa $(\mathrm{m})$ e CC o comprimento de copa $(\mathrm{m})$.

Todas as variáveis apresentaram correlação positiva com o diâmetro à altura do peito, sendo que para as variáveis DC e CC a maior interdependência foi com o DAP.

Mesmo com a verificação de uma grande variabilidade nas dimensões das copas de araucária, o DAP pode ser utilizado em modelo linear simples para descrever o diâmetro de copa, uma vez que este modelo é o mais indicado para explicar a relação seja para árvores de crescimento livre ou para árvores da floresta (Costa et al., 2018).

\section{Competição das copas}

A equação para o diâmetro de copa livre foi $D C L=0,92703+0,15794$ * DAP, a qual apresentou valor para o coeficiente de determinação $\left(R^{2}\right)$ de 0,64 e erro padrão da estimativa de $31,88 \%$. Os resultados quanto à área de copa livre, bem como o FCC em porcentagem para cada parcela, podem ser analisados na Tabela 2.

Tabela 2. Valores da área de copa livre e do fator de competição de copa para cada parcela mensurada do fragmento de Araucaria angustifolia em Lages, SC.

\begin{tabular}{|c|c|c|c|c|c|c|c|}
\hline Parcela & $\sum \mathrm{ACL}$ & $S$ & FCC & Parcela & $\sum \mathrm{ACL}$ & $S$ & FCC \\
\hline 1 & 359,03 & \multirow{13}{*}{400} & 89,76 & 14 & 375,06 & \multirow{13}{*}{400} & 93,77 \\
\hline 2 & 333,25 & & 83,31 & 15 & 576,63 & & 144,16 \\
\hline 3 & 531,62 & & 132,90 & 16 & 571,46 & & 142,86 \\
\hline 4 & 389,23 & & 97,31 & 17 & 344,63 & & 86,16 \\
\hline 5 & 475,84 & & 118,96 & 18 & 150,63 & & 37,66 \\
\hline 6 & 430,18 & & 107,50 & 19 & 446,38 & & 111,59 \\
\hline 7 & 445,94 & & 111,49 & 20 & 292,03 & & 73,00 \\
\hline 8 & 208,67 & & 52,17 & 21 & 382,20 & & 95,55 \\
\hline 9 & 233,78 & & 58,44 & 22 & 347,27 & & 86,82 \\
\hline 10 & 301,39 & & 75,35 & 23 & 448,72 & & 112,18 \\
\hline 11 & 240,92 & & 60,23 & 24 & 327,93 & & 81,98 \\
\hline 12 & 560,09 & & 140,02 & 25 & 520,96 & & 130,24 \\
\hline 13 & 317,01 & & 79,25 & Média & 384,43 & & 96,11 \\
\hline
\end{tabular}

em que: $\sum A C L$ o somatório da área de copa livre da unidade amostral $\left(\mathrm{m}^{2}\right)$, S a área da unidade amostral $\left(\mathrm{m}^{2}\right)$ e $\mathrm{FCC} 0$ fator de competição de copa da unidade amostral (\%). 
Observa-se que a parcela 18 foi a que apresentou o menor valor de FCC $(37,66 \%)$, o que se explica por nessa parcela ter somente duas árvores de araucária. Embora as araucárias dessa parcela tenham uma grande dimensão, com DAP superior a $50 \mathrm{~cm}$ e diâmetros de copa de 10,3 e 9,3 m, suas copas ainda tem espaço para se desenvolverem sem concorrência uma com a outra. Enquanto que, as parcelas 3, 5, 6, 7, 12, 15, 16, 19, 23 e 25 apresentaram valores para o Fator de Competição de Copa maior que 100\%.

Quanto a competição, deve ser destacado que valor 100 para FCC indica que as copas das árvores fecharam completamente o dossel e valores acima sugerem que o espaço não é suficiente para que as copas se desenvolvam, caracterizando uma alta competição entre as mesmas (Schneider \& Schneider, 2008). Essa competição, seja por luz, água e/ou nutrientes é considerada como uma restrição endógena chave no crescimento das árvores, uma vez que tem grande influencia sobre a probabilidade de mortalidade das árvores (Zhang et al., 2017, 2020).

As parcelas que apresentaram valores de FCC acima de 100 se caracterizam por apresentar araucárias que no passado obtiveram um maior desenvolvimento e que agora compõe o dossel da floresta, ou seja, são árvores dominantes. Mesmo que a altura relativa de uma árvore tenha pouca influência na área de projeção da copa, essa variável apresenta grande influência no aumento do comprimento da copa viva das árvores (Forrester et al., 2017), fator que ainda é acentuado quando a altura das árvores vizinhas diminuiu (Thorpe et al. 2010). Esse fator se torna importante para explicar o bom desenvolvimento das árvores de dossel, uma vez que árvores com maiores comprimentos de copa viva promovem maior desenvolvimento. Outra justificativa é que essas árvores se apresentam acima do nível de luz mínimo dentro do dossel que define o ponto de compensação de luz para o desenvolvimento de determinada espécie (Forrester et al., 2017),

Por outro lado, as árvores as árvores que se encontram no subdossel estão limitadas quanto à luminosidade que incide até elas além de uma alteração na sua qualidade (Gonçalves et al., 2010). Isso ocorre pelo fechamento do dossel aliado às condições biológicas da espécie, que possui copas que interceptam aproximadamente $80 \%$ da radiação solar (Seitz, 1976). Essa limitação da luminosidade tem influência negativa para a regeneração de Araucária, uma vez que plantas heliófilas possuem necessidade ecológica de luminosidade para que processo de regeneração natural ocorra normalmente (Avila et al., 2013). Também se ressalta que a disponibilidade de luz nos estratos inferiores das florestas tem importâncias centrais para vários processos do ecossistema florestal (Sercu et al., 2017), afetando o desempenho e a diversidade das plantas do sub-bosque (Bartels; Chen, 2010)

Em termos gerais, quando consideramos a média de FCC de todas as parcelas $(96,11 \%)$, é possível perceber que o dossel desse fragmento se encontra quase que fechado, o que caracteriza uma floresta em competição, corroborando com estudos de Silveira et al. (2018) e Hess et al. (2018a) os quais indicam um superestocamento da área de estudo.

De acordo com Assmann (1970), o espaço médio disponível para o desenvolvimento de uma árvore em uma floresta é inversamente proporcional ao número de árvores por hectare e diretamente proporcional ao DAP dos indivíduos, pois com o aumento do diâmetro das árvores, consequentemente requer um aumento no tamanho médio das copas, requerendo dessa forma, um espaço maior para o crescimento.

Importante enfatizar que deve ser considerado que em povoamentos muito densos, as árvores sofrem intensa competição com as árvores vizinhas, o que levaria a maiores taxas de mortalidade. Essa característica é explicada pois a densidade de árvores é negativamente associada à sobrevivência das mesmas, indicando que as árvores são mais propensas a morrer quando localizadas em locais de maior utilização do espaço (Zhang et al., 2020). Portanto, a estimativa da densidade máxima da floresta, que neste trabalho foi realizada a partir de variáveis de copa, é um requisito essencial para a regulação da densidade (Corral-Rivas et al., 2018).

\section{Determinação da densidade máxima por hectare}

Considerando a alta relação entre essas variáveis, concomitante ao modelo linear ajustado para o diâmetro de copa em função do DAP (Figura 2A), foi modelado o comprimento de copa também 
tendo como variável independente o DAP, onde $C C=2,63978+0,08872 * D A P$, com $R^{2}$ de 0,2698 e Syx\% $=41,88$ conforme apresentado na Figura 2B.

A

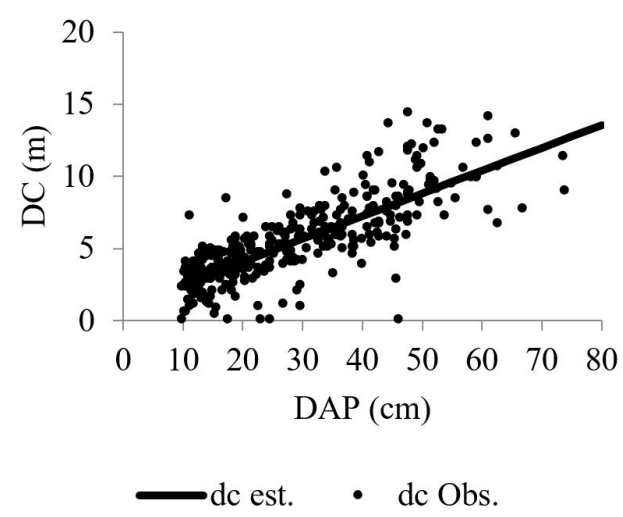

B

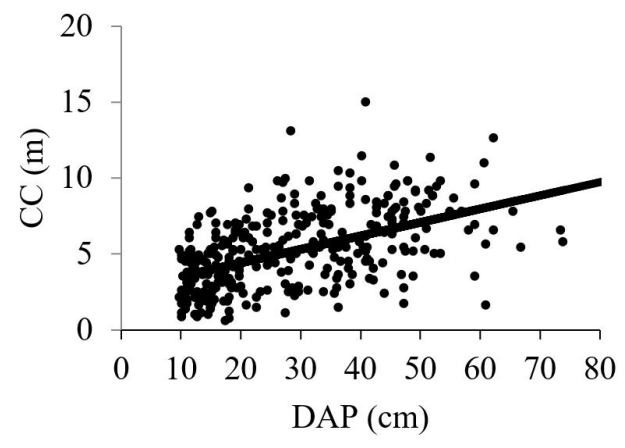

- cc Obs. - $c$ Est.

Figura 2. A) Diâmetro de copa observado (DC Obs) e estimado (DC Est) com a equação ajustada em função do diâmetro a altura do peito (DAP) e B) Comprimento de copa observado (CC Obs) e estimado (CC Est) com a equação ajustada em função do diâmetro a altura do peito (DAP).

Analisando a distribuição gráfica dos resíduos em função dos valores estimados para os modelos ajustados (Figura 3), nota-se que os erros se distribuíram de maneira regular, não apresentando tendências sistemáticas, que superestimassem ou subestimassem os valores.
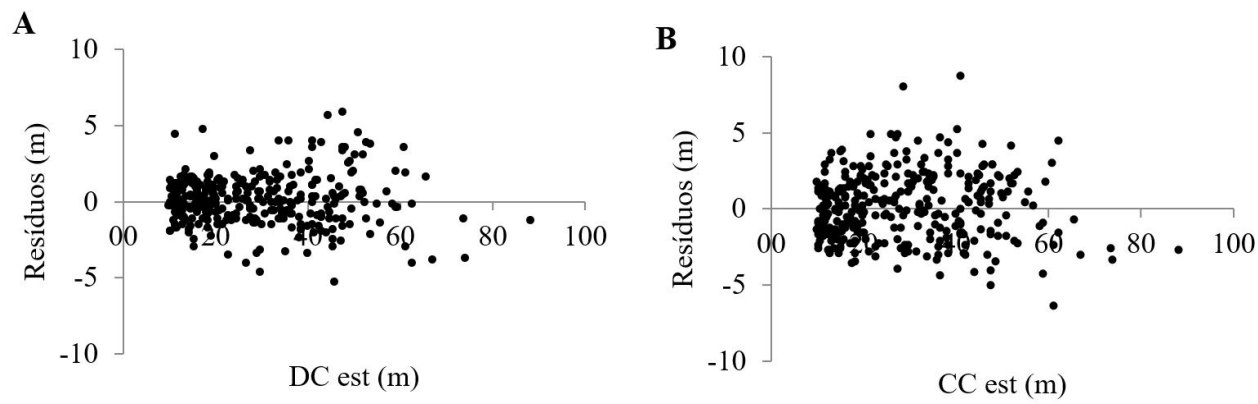

Figura 3. Distribuição dos resíduos para as equações ajustadas para obtenção do diâmetro de copa (DC est) e comprimento de copa (CC est) em função do diâmetro a altura do peito.

A partir do ajuste dos modelos e com base nos diâmetros os quais variaram de 10 a $80 \mathrm{~cm}$, com intervalos de $5 \mathrm{~cm}$ foi possível estimar as variáveis diâmetro de copa e comprimento de copa. Na Tabela 3, observam-se os resultados de diâmetro de copa, comprimento de copa, área de superfície de copa, volume de copa e o número de árvores por hectares em função da área de superfície de copa.

Conforme o DAP aumenta também se observa um aumento da área de superfície de copa e do volume de copa (Tabela 3), resultante da tendência linear positiva entre essas variáveis. Em contrapartida, o número de árvores por hectare tem uma relação negativa com essas variáveis, tendo em vista que quanto maior for a copa da araucária, maior será o espaço requerido pela mesma e assim menor o número de árvores comportado por hectare. Do ponto de vista técnico, seja para produção ou conservação, há necessidade considerar o número médio estimado para essas variáveis, diante da execução da regulação da densidade em área de floresta natural. 
Tabela 3. Diâmetro à altura do peito, diâmetro de copa, comprimento de copa, área de superfície de copa, volume de copa e número de árvores por hectare calculados para um fragmento de Araucaria angustifolia em Lages, SC.

\begin{tabular}{cccccc}
\hline DAP & DC & CC & ASC & VC & N/ha \\
\hline 10 & 2,51 & 3,53 & 14,74 & 2,31 & 679 \\
15 & 3,30 & 3,97 & 22,26 & 3,43 & 449 \\
20 & 4,09 & 4,41 & 31,22 & 4,72 & 320 \\
25 & 4,88 & 4,86 & 41,62 & 6,20 & 240 \\
30 & 5,67 & 5,30 & 53,49 & 7,86 & 187 \\
35 & 6,45 & 5,74 & 66,81 & 9,71 & 150 \\
40 & 7,24 & 6,19 & 81,60 & 11,74 & 123 \\
45 & 8,03 & 6,63 & 97,86 & 13,95 & 102 \\
50 & 8,82 & 7,08 & 115,58 & 16,35 & 87 \\
55 & 9,61 & 7,52 & 134,77 & 18,93 & 74 \\
60 & 10,40 & 7,96 & 155,43 & 21,69 & 64 \\
65 & 11,19 & 8,41 & 177,56 & 24,63 & 56 \\
70 & 11,98 & 8,85 & 201,17 & 27,76 & 50 \\
75 & 12,77 & 9,29 & 226,24 & 31,08 & 44 \\
80 & 13,56 & 9,74 & 252,79 & 34,57 & 40
\end{tabular}

em que: DAP o diâmetro à altura do peito $(\mathrm{cm})$, DC o diâmetro de copa $(\mathrm{m}), \mathrm{CC}$ o comprimento de copa $(\mathrm{m}), \mathrm{ASC}$ a área de superfície de copa $\left(\mathrm{m}^{2}\right)$, VC o volume de copa $\left(\mathrm{m}^{3}\right)$ e $\mathrm{N} / \mathrm{h} a$ o número de árvores por hectare (arv/ha).

Para a definição da densidade máxima de indivíduos por hectare, pode ser utilizada a intersecção das linhas, ou seja, o ponto em que ASC e número de árvores por hectare ou VC e número de árvores por hectare se encontram. Com base nisso e verificando as Figuras 4A e 4B, tem-se no ponto de encontro um diâmetro médio de $32 \mathrm{~cm}$, resultando em um número máximo de 171 árvores por hectare para esse fragmento.

Atualmente, o fragmento de araucária em estudo possui 330 indivíduos por hectare, com um diâmetro médio de $29 \mathrm{~cm}$. Considerando a densidade máxima estabelecida pela ASC ou VC, verifica-se que esse valor se encontra acima do ideal estimado (171 N/ha), embora o diâmetro médio das árvores do fragmento ainda esteja abaixo do calculado. Novamente, esse resultado está de acordo com estudos nessa área de floresta, os quais indicaram que a densidade está acima do ideal para o bom desenvolvimento das árvores presentes no local (Hess et al., 2018a; Silveira et al., 2018).

Através da determinação do número de árvores por hectare considerando o DAP, a ASC e o VC foi possível estabelecer a densidade máxima por hectare para a floresta de araucária (Figuras 4A e 4B). O planejamento da densidade máxima pelas variáveis de copa (ASC e VC) considerando padrões de copa livre (sem competição) busca permitir condições ambientais subótimas para floresta. Portanto, esse planejamento além de considerar um espaço ótimo disponível para expansão lateral da copa, pode estar influenciando na maior disponibilidade de água, luz e nutrientes para as plantas, visto que estima o número de árvores por hectare considerando padrões de crescimento de árvores objetivo.

Com base nas estimativas de densidade seria necessário a redução de $53 \%$ no número de indivíduos por hectare, para que as copas tivessem espaço adequado e suficiente para desenvolverem-se. Considerando que a densidade florestal é um dos principais fatores que influenciam a produção volumétrica (Padilla-Martínez et al., 2020), deve-se salientar que um número de árvores acima do comportado por hectare causa inúmeros efeitos negativos para o desenvolvimento da floresta, como as baixas taxas de incremento devido à competição exercida pelas árvores vizinhas (Aakala et al., 2013; Hess et al., 2018b). Portanto, sugere-se a redução do número de indivíduos por meio do manejo florestal sustentável, a fim de favorecer o crescimento das árvores remanescentes. 
A

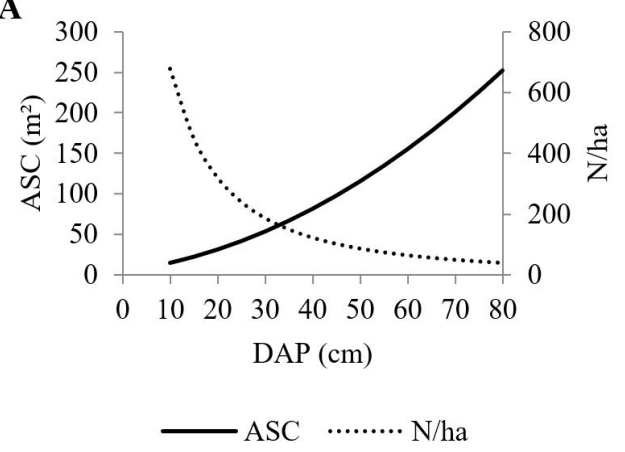

B

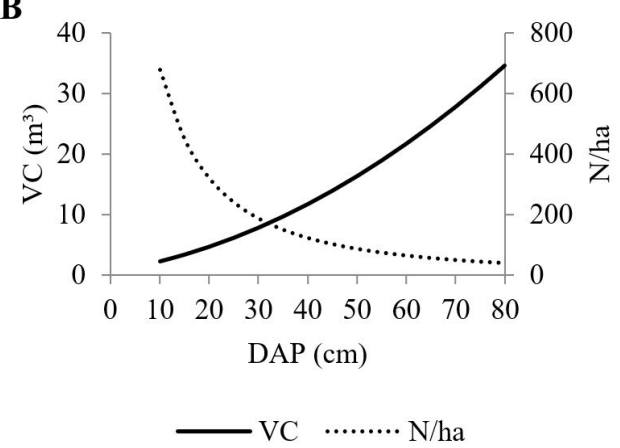

Figura 4. A) Densidade máxima de indivíduos considerando diâmetro a altura do peito (DAP) e as curvas de área de superfície de copa (ASC) e número de árvores por hectare (N/ha) para um fragmento de Araucaria angustifolia em Lages, SC e B) Densidade máxima de indivíduos considerando diâmetro a altura do peito (DAP) e as curvas de volume de copa (VC) e número de árvores por hectare (N/ha) para um fragmento de Araucaria angustifolia em Lages, SC.

Deve-se enfatizar que o conhecimento do potencial produtivo desse ecossistema florestal é essencial para seu uso sustentável (Padilla-Martínez et al., 2020). Assim, pode-se definir quais seriam os pesos das intervenções que seriam realizadas em cada área do ecossistema.

Conforme Curto et al. (2015), a primeira intervenção de indivíduos de araucária deve ser realizada nas classes intermediárias $(30$ a $50 \mathrm{~cm}$ ), tendo em vista que a exploração dessas árvores não prejudicará a questão ecológica do fragmento e ainda possibilitará um retorno financeiro, devido ao seu alto valor comercial. Com a retirada das árvores da classe intermediária, ocorrerá a abertura de clareiras, fato que acelera o incremento das araucárias com menores diâmetros e que permite o desenvolvimento da regeneração natural. Consolidando isso, Reis et al. (2015) destacam que a abertura do dossel em decorrência de intervenção silvicultural de forma sustentável, faz com que tenha um aumento no crescimento das árvores remanescentes.

O manejo florestal sustentável visando o aproveitamento de madeiras de espécies florestais importantes, como é o caso da araucária, torna-se imprescindível para manter as florestas em pé, pois as florestas manejadas vem contribuindo para a maximização da biodiversidade em florestas naturais (Spake et al., 2019) e conservação dos ecossistemas (Chaudhary et al., 2016), uma vez que em áreas protegidas são insuficientes para alcançar as metas globais de conservação da biodiversidade (Laurance et al., 2012).

Contudo, destaca-se a necessidade de utilização de uma boa prática de manejo que vá além das dimensões e a classe social das árvores, mas verificando também a distribuição espacial das árvores remanescentes afim de evitar clareiras extensas e concentração irregular desta vegetação (Curto et al., 2015), pois distúrbios antrópicos sem planejamento são assuntos comumente relacionados com a degradação das florestas (Clark; Covey, 2012).

\section{CONCLUSÃO}

- Todas as variáveis apresentaram correlação positiva com o diâmetro à altura do peito, sendo que as variáveis diâmetro de copa e comprimento de copa apresentaram as maiores correlações, o que justifica a utilização DAP no ajuste das mesmas.

- Dentre as 25 parcelas mensuradas, 10 apresentaram valores para o Fator de Competição de Copa acima de $100 \%$, onde a média para o remanescente foi superior à $95 \%$, indicando que o dossel desse fragmento está quase fechado. Esse fator caracteriza que o mesmo está em competição.

- A área de superfície de copa ou volume de copa possibilitaram definir a densidade máxima de 171 indivíduos por hectare para esse remanescente, com um diâmetro médio de $32 \mathrm{~cm}$, sendo necessário a redução de $53 \%$ no número de indivíduos por hectare, visando um espaço suficiente para o desenvolvimento ideal das copas de araucária. 


\section{AGRADECIMENTOS}

Os autores agradecem ao Fundo de Apoio à Manutenção e ao Desenvolvimento da Educação Superior (FUMDES), a Coordenação de Aperfeiçoamento de Pessoal de Nível Superior (CAPES) e a Fundação de Amparo à Pesquisa e Inovação do Estado De Santa Catarina (FAPESC) pela concessão de bolsa de mestrado.

\section{REFERÊNCIAS}

Aakala, T., Fraver, S., D'Amato, A. W., \& Palik, B. J. (2013). Influence of competition and age on tree growth in structurally complex old-growth forests in northern Minnesota, USA. Forest Ecology and Management, 308, 128-135. http://dx.doi.org/10.1016/j.foreco.2013.07.057.

Alvares, C. A., Stape, J. L., Sentelhas, P. C., de Moraes Gonçalves, J. L., \& Sparovek, G. (2013). Köppen's climate classification map for Brazil. Meteorologische Zeitschrift (Berlin), 22(6), 711-728. http://dx.doi.org/10.1127/0941-2948/2013/0507.

Assmann, E. (1970). The principles of forest yield study (506 p.). Oxford: Ed. Pergamon Press.

Avila, A. L., Araújo, M. M., Gasparin, E., \& Longhi, S. J. (2013). Mecanismos de regeneração natural em remanescente de Floresta Ombrófila Mista, RS, Brasil. Cerne, 19(4), 621-628. http://dx.doi.org/10.1590/S0104-77602013000400012.

Bartels, S. F., \& Chen, H. Y. (2010). Is understory plant species diversity driven by resource quantity or resource heterogeneity? Ecology, 91(7), 1931-1938. PMid:20715612. http://dx.doi.org/10.1890/091376.1.

Burkhart, H. E., \& Tomé, M. (2012). Quantifying Tree Crowns. In H. E. Burkhart \& M. Tomé. Modeling forest trees and stands (458 p.). Heidelberg: Ed. Springer Netherlands. http://dx.doi.org/10.1007/97890-481-3170-9_5.

Chaudhary, A., Burivalova, Z., Koh, L. P., \& Hellweg, S. (2016). Impact of forest management on species richness: global meta-analysis and economic trade-offs. Scientific reports, 6(23954), 23954. PMid:27040604.

Clark, J. A., \& Covey, R. K. (2012). Tree species richness and the logging of natural forests: a metaanalysis. Forest Ecology and Management, Amsterdam, 276, 146-153. http://dx.doi.org/10.1016/j.foreco.2012.04.001

Corral-Rivas, J. J., González-Elizondo, M. S., Lujan-Soto, J. E., \& Gadow, K. V. (2018). Effects of density and structure on production in the communal forests of the Mexican Sierra Madre Occidental. Southern Forests, 81(1), 1-10. http://dx.doi.org/10.2989/20702620.2018.1463152.

Costa, E. A., Finger, C. A. G., \& Fleig, F. D. (2018). Modelagem do espaço de crescimento para araucária. Ciência Florestal, 28(2), 725-734. http://dx.doi.org/10.5902/1980509832085.

Curto, R. A., Zachow, R., Braz, E. M., Mattos, P. P., \& Péllico Netto, S. (2015). Alternativas para o manejo da Floresta Nacional de Açungui (Documentos, 30 p.). Colombo: Embrapa Florestas.

Forrester, D. I., Benneter, A., Bouriaud, O., \& Bauhus, J. (2017). Diversity and competition influence tree allometric relationships - developing functions for mixed-species forests. Journal of Ecology, 105(3), 761-774. http://dx.doi.org/10.1111/1365-2745.12704.

Gonçalves, D. D. A., Schwartz, G., Pokorny, B., \& Van Eldik, T. (2010). O uso da classificação de copa de Dawkins como indicador do comportamento ecológico de espécies arbóreas tropicais. Floresta, 40(1), 175-182. http://dx.doi.org/10.5380/rf.v40i1.17126.

Hess, A. F., Loiola, T., Minatti, M., Rosa, G., Souza, I., Costa, E. A., Schorr, L. P. B., Borsoi, G. A., \& Stepka, T. (2018a). Morphometric Relationships as Indicative of Silvicultural Interventions for Brazilian Pine in Southern Brazil. The Journal of Agricultural Science, 10(7)

Hess, A. F., Ricken, P., \& Ciarnoschi, L. D. (2018b). Dendrocronologia, incremento e manejo florestal em floresta de Araucária-SC. Ciência Florestal, 28(4), 1568-1582. http://dx.doi.org/10.5902/1980509835104.

Hess, A. F., da Silveira, A. C., Krefta, S. M., dos Santos, D. V., Vieira Filho, M. D. H., Atanazio, K. A., Schorr, L. P. B., Souza, I. A., Borsoi, G. A., Stepka, T. F., Costa, E. A., \& Liesenberg, V. (2018c). Crown dynamics of Brazilian pine (Araucaria angustifolia) in Santa Catarina region of Brazil. Australian Journal of Crop Science, 12(3), 449-457. http://dx.doi.org/10.21475/ajcs.18.12.03.pne928.

Kanieski, M. R., Santos, T. L., Graf Neto, J., Souza, T., Galvão, F., \& Roderjan, C. V. (2012). Influência da precipitação e da temperatura no incremento diamétrico de espécies florestais aluviais em Araucária-PR. Floresta e Ambiente, 19(1), 17-25. http://dx.doi.org/10.4322/floram.2012.003. 
Klein, D. R., Hess, A. F., Krefta, S. M., Vieira Filho, M. D. H., Ciarnoscki, L. D., \& Costa, E. A. (2017). Relações morfométricas para Araucaria angustifolia (Bertol.) Kuntze em Santa Catarina. Floresta, 47(4), 501 512. http://dx.doi.org/10.5380/rf.v47i4.49667.

Laurance, W. F., Useche, D. C., Rendeiro, J., Kalka, M., Bradshaw, C. J., Sloan, S. P., Laurance, S. G., Campbell, M., Abernethy, K., Alvarez, P., Arroyo-Rodriguez, V., Ashton, P., Benítez-Malvido, J., Blom, A., Bobo, K. S., Cannon, C. H., Cao, M., Carroll, R., Chapman, C., Coates, R., Cords, M., Danielsen, F., De Dijn, B., Dinerstein, E., Donnelly, M. A., Edwards, D., Edwards, F., Farwig, N., Fashing, P., Forget, P. M., Foster, M., Gale, G., Harris, D., Harrison, R., Hart, J., Karpanty, S., Kress, W. J., Krishnaswamy, J., Logsdon, W., Lovett, J., Magnusson, W., Maisels, F., Marshall, A. R., McClearn, D., Mudappa, D., Nielsen, M. R., Pearson, R., Pitman, N., van der Ploeg, J., Plumptre, A., Poulsen, J., Quesada, M., Rainey, H., Robinson, D., Roetgers, C., Rovero, F., Scatena, F., Schulze, C., Sheil, D., Struhsaker, T., Terborgh, J., Thomas, D., Timm, R., Urbina-Cardona, J. N., Vasudevan, K., Wright, S. J., Arias-G, J. C., Arroyo, L., Ashton, M., Auzel, P., Babaasa, D., Babweteera, F., Baker, P., Banki, O., Bass, M., Bila-Isia, I., Blake, S., Brockelman, W., Brokaw, N., Brühl, C. A., Bunyavejchewin, S., Chao, J. T., Chave, J., Chellam, R., Clark, C. J., Clavijo, J., Congdon, R., Corlett, R., Dattaraja, H. S., Dave, C., Davies, G., Beisiegel, B. M., da Silva, R. N., Di Fiore, A., Diesmos, A., Dirzo, R., Doran-Sheehy, D., Eaton, M., Emmons, L., Estrada, A., Ewango, C., Fedigan, L., Feer, F., Fruth, B., Willis, J. G., Goodale, U., Goodman, S., Guix, J. C., Guthiga, P., Haber, W., Hamer, K., Herbinger, I., Hill, J., Huang, Z., Sun, I. F., Ickes, K., Itoh, A., Ivanauskas, N., Jackes, B., Janovec, J., Janzen, D., Jiangming, M., Jin, C., Jones, T., Justiniano, H., Kalko, E., Kasangaki, A., Killeen, T., King, H. B., Klop, E., Knott, C., Koné, I., Kudavidanage, E., Ribeiro, J. L., Lattke, J., Laval, R., Lawton, R., Leal, M., Leighton, M., Lentino, M., Leonel, C., Lindsell, J., Ling-Ling, L., Linsenmair, K. E., Losos, E., Lugo, A., Lwanga, J., Mack, A. L., Martins, M., McGraw, W. S., McNab, R., Montag, L., Thompson, J. M., Nabe-Nielsen, J., Nakagawa, M., Nepal, S., Norconk, M., Novotny, V., O'Donnell, S., Opiang, M., Ouboter, P., Parker, K., Parthasarathy, N., Pisciotta, K., Prawiradilaga, D., Pringle, C., Rajathurai, S., Reichard, U., Reinartz, G., Renton, K., Reynolds, G., Reynolds, V., Riley, E., Rödel, M. O., Rothman, J., Round, P., Sakai, S., Sanaiotti, T., Savini, T., Schaab, G., Seidensticker, J., Siaka, A., Silman, M. R., Smith, T. B., de Almeida, S. S., Sodhi, N., Stanford, C., Stewart, K., Stokes, E., Stoner, K. E., Sukumar, R., Surbeck, M., Tobler, M., Tscharntke, T., Turkalo, A., Umapathy, G., van Weerd, M., Rivera, J. V., Venkataraman, M., Venn, L., Verea, C., de Castilho, C. V., Waltert, M., Wang, B., Watts, D., Weber, W., West, P., Whitacre, D., Whitney, K., Wilkie, D., Williams, S., Wright, D. D., Wright, P., Xiankai, L., Yonzon, P., \& Zamzani, F. (2012). Averting biodiversity collapse in tropical forest protected areas. Nature, 489(7415), 290-294. PMid:22832582. http://dx.doi.org/10.1038/nature11318.

MacFarlane, D. W., \& Kane, B. (2017). Neighbour effects on tree architecture: functional trade-offs balancing crown competitiveness with wind resistance. Functional Ecology, 31(8), 1624-1636. http://dx.doi.org/10.1111/1365-2435.12865.

Meyer, E. A., Fleig, F. D., Pereira, L. D., \& Vuaden, E. (2013). Ajuste do modelo de Reineke para estimativa da linha de máxima densidade na Floresta Estacional Decidual no Rio Grande do Sul. Revista Árvore, 37(4), 669-678. http://dx.doi.org/10.1590/S0100-67622013000400010.

Narvaes, I. S., Brena, D. A., \& Longhi, S. J. (2005). Estrutura da regeneração natural em Floresta Ombrófila Mista na Floresta Nacional de São Francisco de Paula, RS. Ciência Florestal, 15(4), 331-342. http://dx.doi.org/10.5902/198050981871.

Padilla-Martínez, J. R., Corral-Rivas, J. J., Reyes, J. B., Paul, C., Serrano, M. L., \& Gadow, K. V. (2020). Patterns of Density and Production in the Community Forests of the Sierra Madre Occidental, Mexico. Forests, 11(3), 307. http://dx.doi.org/10.3390/f11030307.

Pretzsch, H. (2014). Canopy space filling and tree crown morphology in mixed-species stands compared with monocultures. Forest Ecology and Management, 327, 251-264. http://dx.doi.org/10.1016/j.foreco.2014.04.027.

Reis, L. P., Reis, P. C. M., Ruschel, A. R., Silva, J. N. M., Carvalho, J. O. P., Souza, A. L., Soares, M. H. M., \& Miyahara, R. K. N. (2015). Forest dynamics in the eastern Amazon with special reference to Sapotaceae species. Floresta, 45(3), 567 - 576. http://dx.doi.org/10.5380/rf.v45i3.35947.

Schneider, P. R., \& Schneider, P. S. P. (2008). Introdução ao Manejo Florestal (2. ed., 566 p.). Santa Maria: Ed. FACOS-UFSM.

Schorr, L. P. B. (2019). Dinâmica e relações alométricas para espécies arbóreas em floresta ombrófila mista sob regime de não-manejo no sul do Brasil (Dissertação de mestrado). Universidade do Estado de Santa Catarina, Lages.

Seitz, R. A. (1976). Estudo da variação da radiação solar, temperatura e umidade relativa do ar no interior de uma mata de Araucaria angustifolia em relação ao terreno livre. Floresta, 7, 36-45. 
Sercu, B. K., Baeten, L., van Coillie, F., Martel, A., Lens, L., Verheyen, K., \& Bonte, D. (2017). How tree species identity and diversity affect light transmittance to the understory in mature temperate forests. Ecology and Evolution, 7(24), 10861-10870. PMid:29299264. http://dx.doi.org/10.1002/ece3.3528.

Silveira, A. C., Hess, A. F., Schorr, L. P. B., Krefta, S. M., Santos, D. V., Filho, M. D. H. V., Atanazio, K. A. A., Costa, E. A., Stepka, T. F., \& Borsoi, G. A. (2018). Management of Brazilian pine (Araucaria angustifolia (Bertol) Kuntze) based on the Liocourt model in a mixed ombrophilous forest in Southern Brazil. Australian Journal of Crop Science, 12(2), 311-317. http://dx.doi.org/10.21475/ajcs.18.12.02.pne927.

Spake, R., Yanou, S., Yamaura, Y., Kawamura, K., Kitayama, K., \& Doncaster, C. P. (2019). Meta-analysis of management effects on biodiversity in plantation and secondary forests of Japan. Conservation Science and Practice, 1(3), e14. http://dx.doi.org/10.1111/csp2.14.

Thomas, C. (2009). Dendrograma de manejo da densidade para povoamentos de Pinus elliottii Engelm na região central do Rio Grande do Sul (Dissertação de mestrado). Universidade Federal de Santa Maria, Santa Maria.

Thorpe, H. C., Astrup, R., Trowbridge, A., \& Coates, K. D. (2010). Competition and tree crowns: a neighborhood analysis of three boreal tree species. Forest Ecology and Management, 259(8), 15861596. http://dx.doi.org/10.1016/j.foreco.2010.01.035.

Zhang, X., Cao, Q. V., Duan, A., \& Zhang, J. (2017). Modeling tree mortality in relation to climate, initial planting density, and competition in Chinese fir plantations using a Bayesian logistic multilevel method. Canadian Journal of Forest Research, 47(9), 1278-1285. http://dx.doi.org/10.1139/cjfr-2017-0215.

Zhang, X., Wang, Z., Chhin, S., Wang, H., Duan, A., \& Zhang, J. (2020). Relative contributions of competition, stand structure, age, and climate $T$ factors to tree mortality of Chinese fir plantations: long-term spacing trials in southern China. Forest Ecology and Management, 465, 118103. http://dx.doi.org/10.1016/j.foreco.2020.118103.

Contribuição dos Autores: ACS: Escrita - Primeira Redação, Investigação, Curadoria de Dados, Validação, Metodologia; AFH: Análise Formal, Supervisão, Metodologia, Conceituação; LPBS: Escrita - Revisão e Edição, I Investigação; TFS: Supervisão, Investigação; SMK e KAA: Investigação, Análise Formal. 